\title{
Consumo e tempo diário de pastejo por novilhos Nelore em pastagem de capim-tanzânia sob diferentes ofertas de forragem
}

\author{
Miguel Marques Gontijo Neto², Valéria Pacheco Batista Euclides ${ }^{3}$, Domicio do Nascimento \\ Júnior $^{4}$, Lídia Ferreira Miranda ${ }^{5}$, Dilermando Miranda da Fonseca ${ }^{6}$, Marcelo Paschoal de Oliveira ${ }^{3}$ \\ 1 Parte da tese de Doutorado em Zootecnia (UFV) do primeiro autor. Financiada pela Embrapa Gado de Corte/CNPq. \\ 2 Embrapa Milho e Sorgo, Cx. Postal 151, CEP: 35701-970, Sete Lagoas, MG. \\ ${ }^{3}$ Embrapa Gado de Corte, Cx. Postal 154, CEP: 79002-970, Campo Grande, MS \\ ${ }^{4}$ Depto. de Zootecnia da UFV, CEP: 36571-000, Viçosa, MG. \\ 5 FEAD-Minas, Belo Horizonte, MG
}

RESUMO - Os efeitos de diferentes níveis de oferta de forragem, associados a alterações no dossel induzidas pelo pastejo, sobre o tempo de pastejo e o consumo de forragem por novilhos mantidos em pastagem de capim-tanzânia foram avaliados neste experimento. Os quatro níveis planejados de oferta de forragem (OF) ( $\mathrm{kg}$ de MS de lâmina foliar/100 $\mathrm{kg}$ de PV animal/dia, \%) resultaram em OF de $6,1 \pm 0,59 ; 11,1 \pm 0,77 ; 18,0 \pm 1,24 ;$ e $23,9 \pm 1,15 \%$. Cada piquete foi pastejado por oito animais Nelore, com peso médio de 229,0 e $249,5 \mathrm{~kg}$, para o primeiro e segundo períodos de amostragem, respectivamente. Foi utilizado um delineamento em blocos casualizados com quatro tratamentos, definidos pelos níveis médios de oferta de forragem. O tempo de pastejo, a disponibilidade de matéria seca de folhas, a relação folha/colmo e a altura do dossel apresentaram alta correlação com o consumo de forragem e podem ser utilizados no desenvolvimento de modelos de predição de consumo de forragem ou desempenho animal em pastejo. Estudos avaliando consumo e desempenho de animais em pastejo em relação a ofertas de forragem necessitam de descrições das disponibilidades e condições estruturais da pastagem para interpretação e comparação de resultados. Alteraçõses nas OF de capim-tanzânia, associadas àquelas nas condições estruturais da pastagem induzidas pelo pastejo tiveram efeito quadrático sobre o tempo diário de pastejo e o consumo de forragem de novilhos Nelore. O menor tempo de pastejo e o maior consumo de forragem foram verificados no nível de OF próximo a $22,5 \mathrm{~kg}$ de lâminas foliares $/ 100 \mathrm{~kg} \mathrm{PV}$, que corresponde a um resíduo pós-pastejo em torno de 4.323,2 kg/ha de MS, $2.887,6 \mathrm{~kg} / \mathrm{ha}$ de matéria verde seca e altura média do dossel de $64 \mathrm{~cm}$.

Palavras-chave: bovinos de corte, gramínea tropical, Panicum maximum, pastejo

\section{Effects of herbage allowance on the intake and grazing time of Nellore steers grazing tanzâniagrass pasture}

\begin{abstract}
This work aimed to evaluate the effects of forage allowances on canopy changes, the grazing time and forage intake by steers grazing tanzaniagrass (Panicum maximum Jacq.) pasture. The four levels of herbage allowance (HA) (kg of leaf blade $/ 100 \mathrm{~kg}$ animal live weight/day, \%) were: $6.1 \pm 0.59 ; 11.1 \pm 0.77 ; 18.0 \pm 1.24$ and $23.9 \pm 1.15 \%$. Eight Nelore animals averaging 229.0 and $249.5 \mathrm{~kg}$ grazed each paddock in the first and second sampling periods, respectively. A completely randomized block design was used. Grazing time, leaf dry matter availability, leaf:stem ratio and canopy height were highly correlated with forage intake and can be used to develop prediction models of forage intake and performance of the grazing animal. Studies on intake and grazing animal performance in relation to forage allowances should consider the pasture structural traits for data interpretation and comparison. Tanzaniagrass forage allowances induced changes in the pasture structural characteristics and had quadratic effect on the daily grazing time and on the forage intake by Nelore steers. Shortest grazing time and highest forage intake were observed on pasture with herbage allowance of about $22.5 \mathrm{~kg}$ leaf blade $100 \mathrm{~kg} \mathrm{BW}$, which corresponded to a postgrazing mass of $4323.2 \mathrm{~kg} / \mathrm{ha}$ dry matter, $2887.6 \mathrm{~kg} / \mathrm{ha}$ dry green matter and average canopy height of $64 \mathrm{~cm}$.
\end{abstract}

Key Words: beef cattle, tropical grassland, Panicum maximum, grazing

\section{Introdução}

A obtenção de altos ganhos por animal depende da otimização do consumo de forragem, que, em situação de pastejo, é influenciada por múltiplos fatores. Esses fatores podem ser agrupados conforme o ambiente, o animal e a pastagem. Os efeitos sobre o consumo relacionados às características da pastagem podem ser divididos em 'disponibilidade' e 'qualidade' da forragem. A disponibilidade refere-se à quantidade e distribuição da massa de forragem no espaço e afeta a taxa de consumo, por meio de aspectos mecânicos de apreensão e ingestão da forragem pelos 
animais (Arnold, 1981). A qualidade envolve todos os atributos físicos e químicos da forragem, afetando a taxa de consumo diretamente, via seletividade de pastejo (Heady, 1964; Hodgson et al., 1994), e indiretamente, via taxa de processamento do alimento no trato digestivo do animal (Bines, 1971).

Em pastagens tropicais, os efeitos da oferta de forragem, associados a aspectos de altura e estrutura do dossel, sobre o consumo de forragem e seu valor nutritivo têm sido pouco avaliados, entretanto, alguns estudos comprovam efeito positivo da oferta de forragem sobre a produção de leite ou as taxas de ganho de peso. Boval et al. (2000) constataram, em breve revisão para áreas de clima temperado, que a relação entre oferta de forragem e consumo é curvilínea, sugerindo que o máximo de consumo foi alcançado em ofertas de forragem entre 55 e $240 \mathrm{~g} \mathrm{MS} / \mathrm{kg}$ PV.

Em experimentos com animais em pastejo, é extremamente difícil isolar os efeitos de características morfológicas e de estrutura do dossel sobre o consumo e o comportamento animal, visto que a própria variação na pressão de pastejo induz alterações estruturais no dossel. Assim, Burns et al. (1994) ressaltaram a importância de informações sobre as disponibilidades de forragem total e de folhas (e suas densidades volumétricas, em $\mathrm{kg} / \mathrm{ha} / \mathrm{cm}$ ) e a qualidade da dieta para a melhor interpretação dos resultados de consumo e desempenho animal em pastejo. Do mesmo modo, Cosgrove (1997) considera fundamental a descrição da condição do dossel no qual foram avaliados os aspectos do comportamento ingestivo e do consumo de forragem por animais em pastejo.

Longos tempos diários de pastejo, indicativos de dificuldades em satisfazer exigências nutricionais, têm sido verificados em bovinos pastejando forrageiras tropicais, mesmo quando grandes quantidades de forragem estão disponíveis (Stobbs, 1970). A estrutura do dossel destas forrageiras influencia o processo de apreensão de forragem, pois a produção, densidade e altura das plantas variam consideravelmente conforme a espécie e o manejo a que estão submetidas (Stobbs, 1973a).

O consumo diário de forragem é função do tempo de pastejo e da taxa de ingestão de forragem, composta pela taxa e pelo tamanho dos bocado. O tamanho do bocado é fortemente afetado pelas condições da pastagem, sobretudo a altura (Hodgson, 1990). Na prática, a taxa de bocados e o tempo de pastejo freqüentemente aumentam quando o tamanho do bocado diminui, mas estes ajustes no comportamento ingestivo nem sempre são suficientes para impedir a queda no consumo diário de forragem pelos animais em pastejo.
Este experimento foi desenvolvido com o objetivo de avaliar os efeitos de diferentes níveis de oferta de forragem, associados a alterações nas condições do dossel induzidas pelo pastejo, sobre o consumo de forragem e o tempo diário de pastejo por novilhos mantidos em pastagem de capim-tanzânia.

\section{Material e Métodos}

O trabalho foi realizado nas dependências da Embrapa Gado de Corte, em Campo Grande, Mato Grosso do Sul, no período de 19/12/2000 a 07/05/2001. O padrão climático da região é descrito, segundo Köppen, na faixa de transição entre Cfa e Aw tropical úmido. A precipitação total no período avaliado foi de $628,7 \mathrm{~mm}$ e as médias das temperaturas mínima, máxima e média foram de 21,3;29,4 e $25,0^{\circ} \mathrm{C}$, respectivamente.

Utilizou-se uma área total de 4 ha, formada em 1994 com Panicum maximum cv. Tanzânia, dividida em oito piquetes, cada um subdividido em dois subpiquetes de 0,25 ha. O solo na área experimental é classificado como Latossolo Vermelho Escuro distrófico - fase cerradão, com 5,77 de pH, 27,42\% de saturação de bases, $1,58 \mathrm{mg} / \mathrm{dm}^{3}$ de fósforo (P-Mehlich 1) e $78,2 \mathrm{mg} / \mathrm{dm}^{3}$ de potássio. Em novembro de 2000, foram distribuídos a lanço $400 \mathrm{~kg} / \mathrm{ha}$ da fórmula NPK 00-20-20 e $200 \mathrm{~kg} / \mathrm{ha}$ de uréia.

O delineamento estatístico utilizado foi em blocos casualizados (dois), com duas repetições por bloco e quatro tratamentos (Gomes, 1976), que consistiram nos níveis de oferta de forragem (OF) de $6,1 \pm 0,59 ; 11,1 \pm 0,77$; $18,0 \pm 1,24 ;$ e 23,9 $\pm 1,15 \%$ (kg de matéria seca de lâminas foliares $/ 100 \mathrm{~kg}$ de PV animal/dia), com taxa de lotação por tratamento de oito animais Nelore com peso médio de 229,0 e 249,5 kg, no primeiro e segundo períodos de amostragem, respectivamente. Como a lotação animal foi a mesma em todos os tratamentos (oito animais) e a área dos piquetes, igual, o ajuste da oferta de forragem (OF), correspondente aos tratamentos, foi obtido por ajustes na disponibilidade de matéria seca de lâminas foliares por piquete, realizada por meio de pastejo com lotação animal variável, na semana anterior ao período de aplicação efetiva dos tratamentos e da coleta dos dados.

O período experimental (dezembro de 2000 a maio de 2001) foi composto por dois períodos de avaliação (blocos) de aproximadamente 56 dias cada -35 dias para acúmulo de forragem nos piquetes, sete para o ajuste da forragem disponível, conforme os tratamentos pretendidos, e 14 dias para a coleta dos dados, com o primeiro período de amostragem entre os dias 31 de janeiro e 13 de fevereiro de 2001 e o segundo, entre os dias 24 de abril e 07 de maio de 2001. 
Durante o período de amostragem, os animais foram mantidos em apenas um dos subpiquetes de cada piquete, com alternância de utilização entre os subpiquetes, visando manter maior homogeneidade das ofertas de forragem definidas pelos tratamentos. Portanto, o termo piquete (repetição) utilizado neste estudo refere-se ao subpiquete ocupado pelos animais.

Para a estimativa das disponibilidades médias de forragem e das disponibilidades médias de MS de lâminas foliares utilizadas para o cálculo das OF, realizou-se, em cada piquete, a caracterização da pastagem (primeiro e último dia do período de amostragem), que consistiu de 30 medições, com régua graduada, da altura do dossel (duas por quadrado amostrado, considerando-se duas touceiras dentro do quadro). Para a disponibilidade de MS, efetuaram-se cortes de 15 amostras utilizando-se um quadrado de $1 \times 1 \mathrm{~m}$ e corte realizado a $15 \mathrm{~cm}$ de altura do solo. Estas amostras foram divididas em duas subamostras (uma para avaliação da disponibilidade de MS e outra para determinação da porcentagem na MS de lâminas foliares, pseudocaules [bainhas foliares mais colmo] e material morto) que foram secas em estufa com ventilação forçada, por 72 horas a $65^{\circ} \mathrm{C}$, trituradas em moinho tipo Willey - $1 \mathrm{~mm}$ e submetidas a análises laboratoriais.

No sétimo e oitavo dias dos períodos de amostragem, foram utilizados quatro animais fistulados no esôfago, com aproximadamente $350 \mathrm{~kg}$, após jejum de 16 horas, para a coleta da extrusa. Foram coletadas duas amostras/piquete, utilizando-se animais distintos para cada amostragem, de modo que parte das amostras de extrusa esofágica proveniente de cada animal foi seca em estufa $\left(55^{\circ} \mathrm{C}\right.$, por 72 horas), moída e analisada quanto à digestibilidade in vitro da matéria seca (DIVMS), conforme descrito por Tilley \& Terry (1963), e aos teores de PB (AOAC, 1990) e FDN (Goering \& Van Soest, 1970).

O consumo de MS (CMS) foi obtido pela fórmula: $\mathrm{CMS}=$ EF*100/(1-DIVMS), em que CMS éo consumo deMS(kg/dia); EF, a excreção fecal diária (kg/dia); e DIVMS, a digestibilidade in vitro da MS média de cada piquete.

A excreção fecal foi determinada com a utilização de indicador externo (óxido cromo III), ministrado ( $10 \mathrm{~g}$ ) uma vez ao dia, às $16 \mathrm{~h}$, durante 12 dias, de forma que, nos últimos cinco dias, foram coletadas amostras de fezes concomitantemente à aplicação do óxido crômico em cinco animais de cada piquete. As amostras de fezes foram acondicionadas em sacos plásticos e congeladas a $-10^{\circ} \mathrm{C}$, para posterior determinação dos teores de cromo (Willians et al., 1962). A excreção fecal (EF) foi obtida conforme descrito por Smith \& Reid (1955): EF (g/dia) = óxido crômico fornecido (g/dia) / Conc. de óxido crômico nas fezes (g/gMS).
O tempo de pastejo diário (TP) foi computado entre o 9 e e 13 o dias de cada período de amostragem, utilizando-se aparelhos vibracorder com capacidade de registro de 24 horas. Foram utilizados quatro aparelhos, instalados diariamente em dois animais por piquete, realizando-se a troca dos aparelhos entre os animais e a substituição dos cartões de registro de cada aparelho no horário em que os animais eram recolhidos ao curral para dosificação do cromo e coleta de fezes. Quando observado algum comportamento estranho do animal com o aparelho, em relação aos demais, os registros foram descartados.

Os dados referentes à excreção fecal, à DIVMS, ao consumo de MS, PB e FDN e ao tempo de pastejo diário foram submetidos à análise de variância e o efeito da oferta de forragem, interpretado por meio de análise de regressão, utilizando-se os procedimentos GLM e REG (SAS, 1990), segundo o modelo estatístico:

$$
\mathrm{Y}_{\mathrm{ijk}}=\mu+\mathrm{t}_{\mathrm{i}}+\mathrm{b}_{\mathrm{j}}+\mathrm{e}_{\mathrm{ijk}} \text {, }
$$

em que $\mathrm{Y}_{\mathrm{ijk}}=$ valor observado relativo ao tratamento $\mathrm{i}$, ao período $\mathrm{j}$ e à repetição $\mathrm{k} ; \mu=$ média geral; $\mathrm{t}_{\mathrm{i}}=$ efeito do tratamento $i$, sendo $i=1,2,3$ e $4 ; b_{j}=$ efeito do período ou bloco $\mathrm{j}$, sendo $\mathrm{j}=1,2$ e 3 ; e $\mathrm{e}_{\mathrm{ijk}}=$ erro aleatório associado a cada observação, sendo $\mathrm{k}=1$ e 2 .

Os modelos de regressão foram escolhidos com base na significância da regressão e da falta de ajustamento, testadas pelo teste F, na significância dos coeficientes de regressão, utilizando-se o teste $\mathrm{t}$ de Student, e no coeficiente de determinação. Foi adotado nível de significância de até 5\% de probabilidade, em que * representa significância a 5\% e ** significância a $1 \%$ pelo teste t de student. Foram também determinadas correlações simples, aplicando-se o teste de Pearson a 5 e $1 \%$ de probabilidade.

\section{Resultados e Discussão}

Em função da OF, em kg de MS de lâminas foliares $/ 100 \mathrm{~kg}$ de peso vivo animal (\%), a excreção fecal (EF, kg de MS/an/ dia) apresentou comportamento quadrático $(E F=1,0124+$ $\left.0,0587^{* *} \mathrm{OF}-0,0038^{* *} \mathrm{OF}^{2} ; \mathrm{R}^{2}=0,9939\right)$, com valores de 1,84 a $2,63 \mathrm{~kg} / \mathrm{an} /$ dia e valor máximo $(2,67 \mathrm{~kg} / \mathrm{an} / \mathrm{dia})$ obtido com OF de $20,9 \%$.

Da mesma forma, a DIVMS também apresentou comportamento quadrático em decorrência da OF (DIVMS $=37,6487$ $\left.+1,5477 * * \mathrm{OF}-0,0351 * * \mathrm{OF}^{2} ; \mathrm{R}^{2}=0,9985\right)$, com valores estimados entre 45,8 e $54,7 \%$, sendo que a diferença de $8,9 \%$ entre o maior e menor valor se deve às variações nas proporções de folha, colmos e material morto na dieta, visto que os animais selecionaram os componentes de melhor qualidade com o aumento da OF (Gontijo Neto, 2003). 
As equações ajustadas para os consumos de MS (CMS, kg/an/dia) e de MS em relação ao PV animal (CPVMS, $\mathrm{kg} / 100 \mathrm{~kg} \mathrm{PV}$ ), de acordo com as OF avaliadas, encontram-se na Tabela 1 e Figura 1. O CMS variou entre 3,39 e 5,88 kg de MS/an/dia e o CPVMS, entre 1,43 e 2,44\% para as OF avaliadas.

Os valores de OF que propiciaram CMS e CPVMS máximos de 22,03 e 22,19\%, respectivamente (aparentemente elevados em se tratando de oferta de folhas), não devem ser considerados isoladamente, pois devem ser observados os aspectos estruturais da pastagem. Portanto, para os menores e maiores CMS e CPVMS, a altura média do dossel (ALT) foi de 26,9 e 62,3 cm, enquanto a disponibilidade de matéria seca total (MST) correspondeu a 2.190,3 e 4.272,4 kg/ha; a de matéria verde seca(MVS), a 1.140,8 e 2.846,1 kg/ha; de matéria seca de folhas (MSF), a 4.60,6 e 1.673,1 kg/ha; e a relação folha/colmo (F/C), a 0,44 e 1,15, respectivamente, demonstrando enorme amplitude dos valores referentes às características da pastagem. Nas condições deste experimento, as variáveis ALT, MST, MVS, MSF e F/C, relacionadas ao consumo e ao comportamento ingestivo dos animais, apresentaram decréscimos com a redução na OF (Figura 1), o que provavelmente dificultou a seleção da dieta e apreensão de forragem pelos animais, resultando no decréscimo do consumo de forragem.

A máxima eficiência de CPVMS (2,42\%) foi próxima a valores obtidos por outros autores em pastagem de capimtanzânia. Euclides et al. (1999), em novilhos sob pastejo contínuo em pastagem de capim-tanzânia, registraram valores de $52,8 \%$ de folhas, MST média de $2.332,9 \mathrm{~kg} / \mathrm{ha}$, MVS média de $1.710,0 \mathrm{~kg} / \mathrm{ha}$ e MSF média de $1.210,0 \mathrm{~kg} / \mathrm{ha}$, no período das águas, e consumo de MS médio em relação ao PV de $2,46 \%$. Valor semelhante de CPVMS (2,35\%) foi relatado por Lima et al. (2001), utilizando pastagens de capimtanzânia sob pastejo rotativo, com três dias de ocupação por vacas em lactação. Esses autores obtiveram disponibilidade de MS de 7.340,2 $\mathrm{kg} \mathrm{MS} / \mathrm{ha}$ antes e $4.555,9 \mathrm{~kg}$

Tabela 1 - Consumos de MS (CMS, kg/an/dia), NDF (CFDN, kg/an/ dia) e PB (CPB, kg/an/dia), conforme a oferta de forragem (OF, \%)

Table 1 - Intakes of DM (CMS, $\mathrm{kg} / \mathrm{an} /$ day), NDF (CFDN, $\mathrm{kg} / \mathrm{an} / \mathrm{day})$ and $C P$ (CPB, kg/an/day), as affected by herbage allowance (OF)

\begin{tabular}{lcc}
\hline $\begin{array}{l}\text { Variável } \\
\text { Variable }\end{array}$ & $\begin{array}{c}\text { Equação ajustada } \\
\text { Adjusted equation }\end{array}$ & $\mathrm{r}^{2} / \mathrm{R}^{2}$ \\
\hline $\begin{array}{l}\mathrm{CMS} \\
(\mathrm{kg} / \mathrm{an} / \mathrm{dia})\end{array}$ & $\hat{\mathrm{Y}}=1,1207+0,4318 * * \mathrm{OF}-0,0098 * * \mathrm{OF}^{2}$ & 0,9986 \\
$\begin{array}{l}\mathrm{CFDN} \\
(\mathrm{kg} / \mathrm{an} / \mathrm{dia})\end{array}$ & $\hat{\mathrm{Y}}=0,9879+0,3128 * * \mathrm{OF}-0,007 * * \mathrm{OF}^{2}$ & 0,9988 \\
$\begin{array}{l}\mathrm{CPB} \\
(\mathrm{kg} / \mathrm{an} / \mathrm{dia})\end{array}$ & $\hat{\mathrm{Y}}=0,1372+0,0131 * * \mathrm{OF}$ & 0,9658 \\
\hline
\end{tabular}

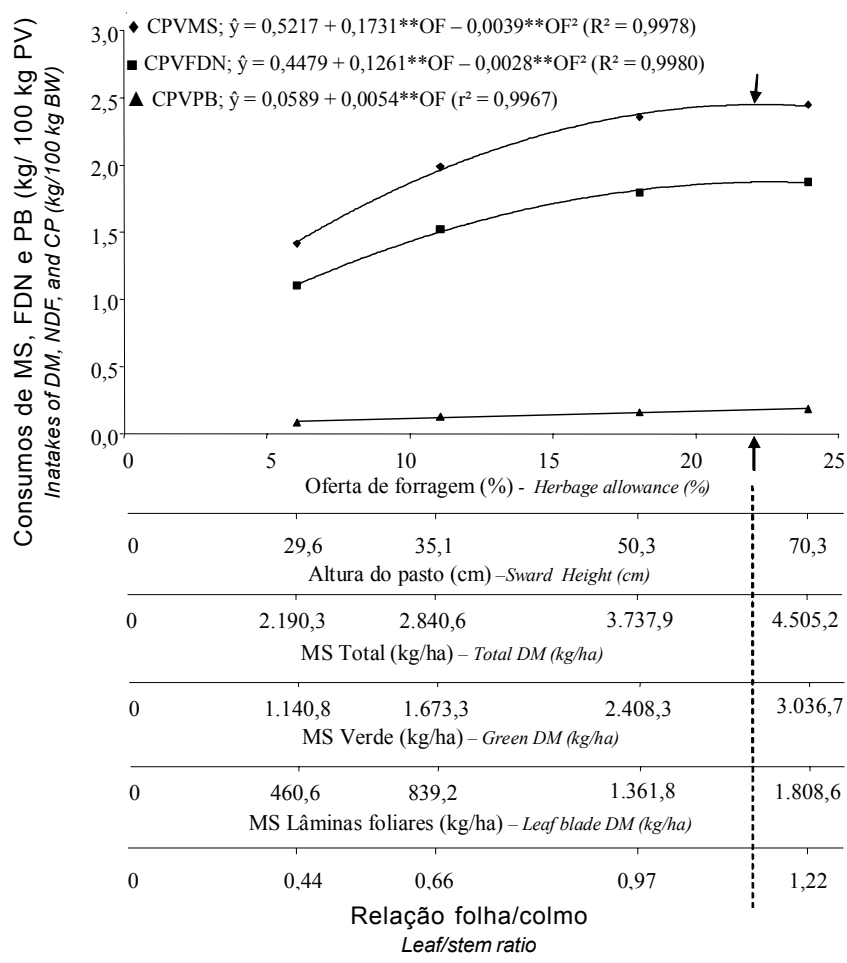

Figura 1 - Consumos de MS (CPVMS, kg/100 kg PV), consumo de FDN (CPVFDN, kg/100 kg PV) e consumo de PB (CPVPB, kg/100 kg PV), em relação ao PV, de acordo com a oferta de forragem (OF) e as características estruturais da pastagem.

Figure 1 - Dry matter intake (CPVMS, $\mathrm{kg} / 100 \mathrm{~kg} \mathrm{BW}$ ), neutral detergent fiber intake (CPVFDN, $\mathrm{kg} / 100 \mathrm{~kg} B W$ ) and crude protein intake (CPVPB, $\mathrm{kg} / 100 \mathrm{~kg} \mathrm{BW}$ ), as affected by herbage allowance (OF) and structural characteristics of the pasture.

MS/ha após o pastejo e disponibilidade de folhas antes e após o pastejo de 5.639,5 e 2.650,6 kg MS de folhas/ha, respectivamente.

Os consumos de $\mathrm{PB}$ (CPB, kg/an./dia) e PB em relação ao $\mathrm{PV}$ animal (CPVPB, \%) apresentaram resposta linear positiva ao aumento da OF (Tabela 1; Figura 1), com valores de CPB entre $0,217 \mathrm{e} 0,450 \mathrm{~kg} / \mathrm{an} . /$ dia e de CPVPB entre $0,092 \mathrm{e} 0,188 \%$ do PV para a menor e a maior OF avaliadas, respectivamente. Segundo o NRC (1996), novilhos com peso em torno de $240 \mathrm{~kg}$ necessitam de, aproximadamente, $0,384 \mathrm{~kg}$ de PB para mantença, que, nas condições deste experimento, foi atingida com OF de $18,84 \%$.

Os consumos de FDN (CFDN, kg/an/dia) e de FDN em relação ao PV animal (CPVFDN, \% do PV) sofreram efeito quadrático das variações na OF (Tabela 1; Figura 1), cujos valores de CFDN foram de 2,63 a 4,48 kg de FDN/animal/ dia, com valores máximos de OF de $22,34 \%$ e de CPVFDN de 1,11 a 1,86 kg de FDN/100 kg de PV/dia, com valor máximo de OF de 22,51\%.

Para OF acima de 7,07\%, o consumo de FDN foi superior a 1,2\% do PV, identificado empiricamente por Mertens (1994) 
como limitante, por ação física, ao consumo de forragem por bovinos. Entretanto, em estudos com forrageiras tropicais, encontram-se com freqüência valores para CPVFDN acima de $1,2 \%$ do PV, confirmando a necessidade de validação ou determinação deste índice para condições tropicais. Euclides et al. (1999), avaliando gramíneas do gênero Panicum (colonião, tobiatã e tanzânia), utilizando extrusas esofágicas, obtiveram valores médios de CPVFDN por novilhos em pastejo de 1,45 e 1,92\% do PV, nos períodos da seca e das chuvas, respectivamente. Lima et al. (2001), em pastagem de tanzânia e extrusas esofágicas, obtiveram valor de CPVFDN de 1,85\% de PV por vacas em lactação em pastejo.

O coeficiente de utilização da forragem, definido neste estudo como a quantidade de MS consumida ( $\mathrm{kg}$ de MS $/ 100 \mathrm{~kg}$ $\mathrm{PV} /$ dia) em relação à MS de lâminas foliares ofertadas ( $\mathrm{kg}$ de MS de lâminas foliares/100 kg PV/dia), apresentou resposta linear inversa à $\mathrm{OF}$ (Figura 2), com valores entre 9,39 e $22,92 \%$ para a maior e menor $\mathrm{OF}$, respectivamente. O incremento verificado no consumo de forragem, de acordo com o aumento da OF (Figura 2), não foi suficiente para manter o nível de utilização da forragem ofertada.

O consumo de forragem e o coeficiente de utilização da forragem ofertada apresentaram tendência semelhante à descrita por Hodgson (1990), ressaltando-se que os baixos índices de utilização da forragem verificados neste estudo,

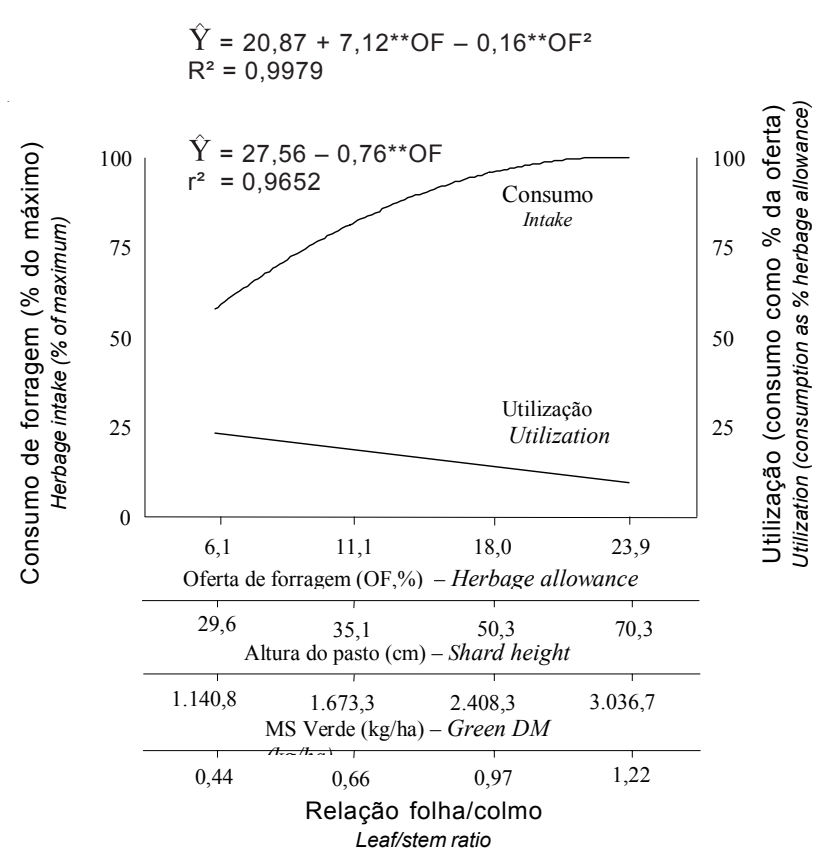

Figura 2 - Consumo de forragem (\% do máximo) e coeficiente de utilização da forragem ofertada (\%), de acordo com a oferta de forragem (\%) e as características estruturais da pastagem.

Figure 2 - Forage intake (\% of the maximum) and coefficient of utilization of the herbage allowance (\%), as affected by herbage allowance (OF) and structural characteristics of the pasture. provavelmente, são decorrentes dos aspectos estruturais da pastagem.

O tempo diário de pastejo $(\mathrm{TP}, \mathrm{h})$ apresentou comportamento quadrático, conforme a variação na OF (Figura 3), com valores entre 6,2 e 10,1 horas, com maiores valores observados para menores OF. Segundo Hodgson (1990), tempos de pastejo acima de 8 a 9 horas por dia podem ser indicativos de condições do relvado limitantes ao consumo de forragem. Nas condições deste experimento, apesar do aumento no TP, decorrente da diminuição da $\mathrm{OF}$, o TP não foi suficiente para impedir o decréscimo do consumo de forragem pelos animais, como conseqüência da redução da taxa de consumo de forragem, resultante de provável diminuição do tamanho do bocado.

O aumento na atividade de pastejo com a diminuição da oferta de forragem foi verificado também por Ribeiro Filho et al. (1997), que, avaliando o tempo de pastejo de bovinos submetidos a diferentes OF de capim-elefante anão cv. Mott (3,8; 7,5; 10,5; e 14,7 kg de MS de LFV/100 kg PV/dia), observaram TP de 5,2 a 9,7 horas e decréscimo no TP de $0,43 \mathrm{~h} / \mathrm{kg}$ de lâmina foliar ofertada/ $100 \mathrm{~kg}$ PV/dia.

Valores semelhantes aos verificados neste estudo foram relatados por Euclides et al. (1999), que, em estudo comparativo de cultivares do gênero Panicum, observaram TP de novilhos em pastagem de tanzânia de 561

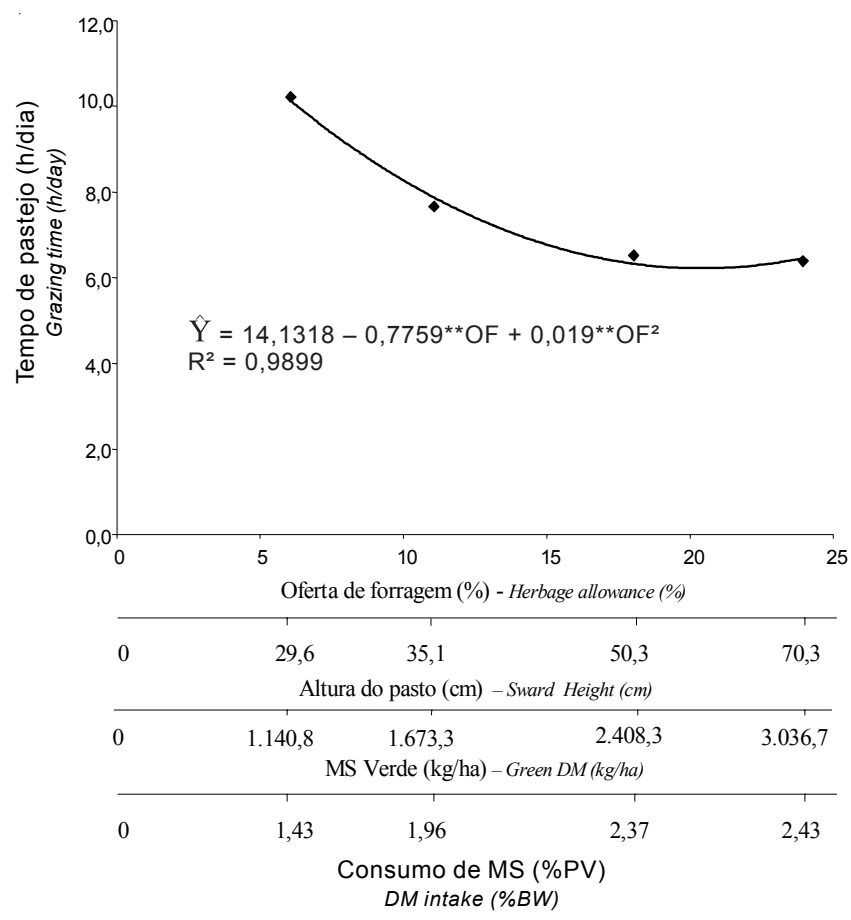

Figura 3 - Tempo de pastejo (h/dia), de acordo com a oferta de forragem (OF, \%), as características estruturais da pastagem e o consumo.

Figure 3 - $\quad$ Time grazing (h/day) affected by herbage allowance (OF, \%) and structural characteristics of the pasture and intake. 
Tabela 2 - Coeficientes de correlação linear entre consumo, tempo de pastejo e características agronômicas e estruturais da pastagem

Table 2 - Coefficients of linear correlation among intake, time grazing and agronomic and structural characteristics of the pasture

\begin{tabular}{|c|c|c|c|}
\hline & $\begin{array}{c}\text { CMS } \\
\text { (kg/an/dia) }\end{array}$ & $\begin{array}{c}\text { CPV } \\
(\mathrm{kg} / 100 \mathrm{~kg} \mathrm{PV})\end{array}$ & $\begin{array}{c}\text { T P } \\
\text { (horas/dia) }\end{array}$ \\
\hline $\mathrm{CMS}^{1}$ (kg/an/dia) & 1 & $0,9737 * *$ & $-0,9593 * *$ \\
\hline $\begin{array}{l}\mathrm{CPV}^{2} \\
(\mathrm{~kg} / 100 \mathrm{~kg} \mathrm{PV})\end{array}$ & $0,9737 * *$ & 1 & $-0,9345 * *$ \\
\hline $\mathrm{TP}^{3}$ (horas/dia) & $-0,9593 * *$ & $-0,9345 * *$ & 1 \\
\hline $\mathrm{ALT}^{4}(\mathrm{~cm})$ & $0,8338 * *$ & $0,8432 * *$ & $-0,8596 * *$ \\
\hline $\mathrm{F} / \mathrm{C}^{5}$ & $0,8895 * *$ & $0,8693 * *$ & $-0,7878 * *$ \\
\hline $\operatorname{MST}^{6}(\mathrm{~kg} / \mathrm{ha})$ & $0,9094 * *$ & $0,9032 * *$ & $-0,8470 * *$ \\
\hline $\operatorname{MVS}^{7}$ (kg/ha) & $0,9194 * *$ & $0,9293 * *$ & $-0,8542 * *$ \\
\hline $\operatorname{MSF}^{8}$ (kg/ha) & $0,9229 * *$ & $0,9245 * *$ & $-0,8660 * *$ \\
\hline
\end{tabular}

${ }^{1} \mathrm{CMS}$ - consumo de matéria seca (kg/an./dia); ${ }^{2} \mathrm{CPV}$ - relação entre a ingestão de matéria seca e o peso vivo animal ( $\mathrm{kg}$ de MS/100 kg PV); ${ }^{3} \mathrm{TP}$ - tempo de pastejo diário (horas/dia); ${ }^{4} \mathrm{ALT}$ - altura $(\mathrm{cm}) ;{ }^{5} \mathrm{~F} / \mathrm{C}$ - relação folha/colmo da forragem; ${ }^{6} \mathrm{MST}$ - matéria seca total disponível (kg/ha); ${ }^{7} \mathrm{MVS}$ - matéria verde seca disponível (kg/ha); ${ }^{8} \mathrm{~V} / \mathrm{T}$ - relação matéria seca verde/matéria seca total.

${ }^{1} \mathrm{CMS}$ - dry matter intake ( $\left.\mathrm{kg} / \mathrm{an} . / \mathrm{day}\right) ;{ }^{2} \mathrm{CPV}$ - dry matter intake ( $\mathrm{kg}$ of DM/100kg BW): ${ }^{3} \mathrm{TP}$ - daily time grazing (hours/day): ${ }^{4} \mathrm{ALT}$ - sward height $(\mathrm{cm}):{ }^{5} \mathrm{~F} / \mathrm{C}$ - forage leaf/stem ratio; ${ }^{6} \mathrm{MST}$ - total dry matter $(\mathrm{kg} / \mathrm{ha}) ;{ }^{7} \mathrm{MVS}$ - green dry matter $(\mathrm{kg} / \mathrm{ha}) ;{ }^{8} \mathrm{~V} / \mathrm{T}$ - green dry matter/ total dry matterratio.

minutos (9,35 h), enquanto Brâncio (2000) registrou valores médios entre 8,7 e 10,2 horas, também com novilhos em pastagem de capim-tanzânia.

Quanto às correlações lineares verificadas entre as características da pastagem, o consumo de forragem foi positivamente correlacionado $(\mathrm{P}<0,01)$ às disponibilidades de forragem total, de MVS e de folhas, à relação folha/colmo e à altura e negativamente correlacionado ao tempo de pastejo. OTP, por sua vez, foi negativamente correlacionado a todas essas características da pastagem (Tabela 2). Essas correlações foram também verificadas por Euclides et al. (1999), à exceção da disponibilidade MS total, em pastagem de tanzânia.

Ressalta-se que o TP apresentou elevadas correlações negativas com o CMS e CPV, podendo ser uma variável interessante, representativa do comportamento animal frente às alterações nas condições da pastagem a ser considerada no desenvolvimento de modelos de predição de consumo e desempenho por animais pastejando forrageiras tropicais, juntamente ao valor nutritivo da forragem e às características estruturais da pastagem.

\section{Conclusões}

O tempo de pastejo, a disponibilidade de MS de folhas, a relação folha/colmo e a altura do dossel apresentaram-se altamente correlacionados ao consumo de forragem e podem ser utilizados no desenvolvimento de modelos de predição de consumo de forragem ou desempenho animal em pastejo.
Estudos para avaliação do consumo e desempenho de animais em pastejo em relação a ofertas de forragem necessitam de descrições das disponibilidades e condições estruturais da pastagem para interpretação e comparação de resultados.

Alterações na oferta de forragem de capim-tanzânia, associadas a alterações nas condições estruturais da pastagem, induzidas pelo pastejo, influenciaram o consumo de forragem e o comportamento animal, de modo que maiores consumos de forragem e menores tempos de pastejo diário foram verificados com $4.258,1 \mathrm{~kg} / \mathrm{ha}$ de MS de resíduo póspastejo, $2.834,4 \mathrm{~kg} / \mathrm{ha}$ de matéria verde seca e $62,3 \mathrm{~cm}$ de altura média do dossel.

Em sistemas de produção utilizando o capim-tanzânia sob pastejo rotativo, estes valores podem ser utilizados como referenciais durante o período de utilização, abaixo dos quais o desempenho animal tenderia a reduzir, indicando a necessidade de adoção de práticas de manejo por parte do produtor, como a utilização de animais repassadores ou a suplementação alimentar, visando otimizar os ganhos por animal e por área.

\section{Literatura Citada}

ARNOLD, G.W. Grazing behaviour. In: MORLEY, F.H.W. (Ed.) Grazing animals. Amsterdam: Elsevier Scientific, 1981. 411p. ASSOCIATION OF OFFICIAL ANALYTICAL CHEMISTS - AOAC. Official methods of analyses. 15.ed. 1990. v.1, p.72-74.

BINES, J.A. Metabolic and physical control of food intake in ruminants. Proceedings of Nutrition Society, v.30, p.116-122, 1971

BOVAL, M.; CRUZ, J.L.; PENNINGS, P.D. The effect of herbage allowance on daily intake by creole heifers tethered on natural Dichanthium ssp. Pasture. Grass and Forage Science, v.55, p.201-208, 2000.

BRÂNCIO, P.A. Comportamento animal e estimativas de consumo por bovinos em pastejo. Viçosa, MG: Universidade Federal de Viçosa, 2000. 268p. Tese (Doutorado em Zootecnia) - Universidade Federal de Viçosa, 2000.

BURNS, J.C.; POND, K.R.; FISHER, D.S. Measurements of forage intake. In: FAHEY JR., G.C. (Ed.). Forage quality, evaluation and utilization. Winsconsin: American Society of Agronomy, 1994. p.494-531.

COSGROVE, G.P. Grazing behaviour and forage intake. In: SIMPÓSIO INTERNACIONAL SOBRE PRODUÇÃO ANIMAL EM PAStejo, 1997, Viçosa, MG. Anais... Viçosa, MG: Universidade Federal de Viçosa, 1997. p.59-80.

EUCLIDES, V.P.B.; THIAGO, L.R.L.; MACEDO, M.C.M. et al. Consumo voluntário de forragem de três cultivares de Panicum maximum sob pastejo. Revista Brasileira de Zootecnia, v.28, n.6, p.1177-1185, 1999 .

GOERING, H.K.; Van SOEST, P.J. Forage fiber analyses, apparatus, reagents, procedures and some applications. Washington, DC: USDA, 1970. (Agricultural Handbook, 379).

GOMES, F.P. Curso de estatística experimental. 6.ed. São Paulo: Nobel, 1976. 430p.

GONTIJO NETO, M.M. Características e qualidade do capimtanzânia (Panicum maximum Jacq.) sob pastejo em diferentes ofertas de forragem. Viçosa, MG: Universidade Federal de Viçosa, 2003. 76p. Tese (Doutorado em Zootecnia) - Universidade Federal de Viçosa, 2003. 
HEADY, H. Palatability of herbage and animal preference. Journal of Range Management, v.17, p.76-82, 1964.

HODGSON, J. Grazing management: science into practice. New York: John Wiley, 1990. 203p.

HOdGSON, J.; CLARCK, D.A.; MITCHELL, R.J. Foraging behaviour in grazing animals and its impact on plant communities. In: FAHEY, G.C. (Ed.) Forage quality, evaluation and utilization. Lincon: American Society of Agronomy, 1994. p.796-827.

LIMA, M.L.P.; BERCHIELli, T.T.; NOGUEIRA, J.R. et al. Estimativa do consumo voluntário do capim-tanzânia (Panicum maximum, Jacq. cv. Tanzânia) por vacas em lactação sob pastejo rotacionado. Revista Brasileira de Zootecnia, v.30, n.6, p.1919-1924, 2001.

MERTENS, D.R. Regulation of forage intake. In: FAHEY JR., G.C. et al. (Eds.). Forage quality evaluation and utilization. Nebraska: American Society of Agronomy, Crop Science of America, Soil Science of America, 1994. 988p.

NATIONAL RESEARCH COUNCIL - NRC. Nutrient requirement of beef cattle. 7.rev.ed. Washington, D.C.: National Academy Press, 1996. 242p.

RIBEIRO FILHO, H.M.N.; ALMEIDA, E.X.; HERTHMANN, O.E.L. et al. Tempo e ciclos diários de pastejo de bovinos submetidos a diferentes ofertas de capim-elefante anão cv. 'Mott'. In: REUNIÃO ANUAL DA SOCIEDADE BRASILEIRA DE ZOOTECNIA, 34., 1997, Juiz de Fora. Anais... Minas Gerais: Sociedade Brasileira de Zootecnia, 1997. p.276-278.

STATISTICAL ANALYSES SYSTEM - SAS. SAS/STAT. User's guide. Version 6. 4.ed. Cary: 1999. 846p.
SMITH, A.M.; REID, J.T. Use of chromic oxide as an indicator of fecal output for the purpose of determining the intake of a pasture herbage by grazing cows. Journal Dairy Science, v. 38 , n.5, p.515-524, 1955.

STOBBS, T.H. Automatic measurement of the grazing time by diary cows on tropical grass and legume pastures. Tropical Grassland, v.4, n.3, p.237-244, 1970.

STOBBS, T.H. The effect of plant structure on the intake of tropical pastures. I. Variation in the bites size of the grazing cattle. Australian Journal of Agricultural Research, v.24, n.6, p.809-819, 1973.

TILLEY, J.M.A.; TERRY, R.A. A two stage technique for the in vitro digestion of forage crops. Journal of the British Grassland Society, v.18, n.2, p.104-111, 1963.

WILLIAMS, C.H.; DAVID, D.J.; IISMAA, O. The determination of chromic oxide in feces samples by atomic absorption spectrophotometry. Journal of Agricultural Science, v.59, n.3, p.381-385, 1962 . 